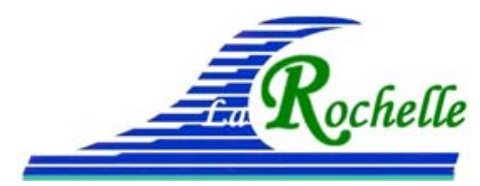

XVèmes Journées Nationales Génie Côtier - Génie Civil

La Rochelle, 29 au 31 mai 2018

DOI:10.5150/jngcgc.2018.026

(C) Editions Paralia CFL

disponible en ligne - http://www.paralia.fr - available online

\title{
Modélisation du transport des sédiments fins à l'embouchure du fleuve Bandama ; Grand-Lahou, Côte d'Ivoire
}

\author{
Kouakou Lazare KOUASSI ${ }^{\text {, }}$ Kouakou Séraphin KONAN ${ }^{1}$, \\ Martin SANCHEZ ${ }^{2}$, KouaméAKA ${ }^{3}$
}

1. Université Jean Lorougnon Guédé, BP 150 Daloa (Côte d'Ivoire) ; k_lazare@yahoo.fr

2. Laboratoire de Planétologie et Géodynamique, Université de Nantes, Faculté des Sciences et des Techniques. martin.sanchez@univ-nantes.fr

3. Université Félix-HOUPHOUËT-BOIGNY, Abidjan, Côte d'Ivoire, akaraphael@yahoo.fr

\section{Résumé :}

L'estuaire de Grand-Lahou représente, de par sa diversité écologique et environnementale, une importante source de subsistance et une source de revenus pour les populations riveraines dont la principale activité économique est la pêche traditionnelle. Cependant, l'érosion côtière constitue une réelle menace sur le littoral ivoirien et particulièrement à l'embouchure du fleuve Bandama. La dynamique hydrosédimentaire a entrainé le déplacement de la ville de Grand-Lahou dans la partie nord de cette région dans les années 70. L'étude des phénomènes hydrosédimentaires dans cette zone s'avère nécessaire pour comprendre l'évolution de la morphologie du fond du système lagunaire de Grand-Lahou et évaluer l'impact des mouvements sédimentaires sur les chenaux de navigation et le port de pêche. Ainsi, une modélisation du transport des sédiments a été entreprise dans la lagune avec le modèle MIKE 21 FM, en couplant le module MT (Mud Transport) au module hydrodynamique HD afin de simuler la dynamique des sédiments vaseux et des sables fins qui se fait principalement en suspension dans la colonne d'eau. Les résultats montrent que les fonds vaseux sont situés dans les secteurs Est et Ouest de la lagune, ainsi qu'à quelques endroits le long de la rive Nord et sur le bord du chenal de Tagba. En période d'étiage, les vases tendent à se déposer dans les chenaux du Bandama et de Lahou-Kpanda, alors qu'elles sont évacuées en période de crue. La dynamique des sables fins est limitée au secteur central en période d'étiage, soumis à l'action des courants de marée. En période de crue, les forts courants tendent à éroder les chenaux du Bandama et de Lahou-Kpanda, générant un transport vers l'Ouest. On observe une tendance à l'accumulation au Sud du port de pêche et à la flèche de Brafedon. Dans le secteur de la passe, sont surtout présents les sables moyens du fait d'un hydrodynamisme fort. Le modèle permet de reproduire la dynamique des vases et des sables fins dans la lagune sous l'effet de la marée et de différentes conditions hydrologiques du Bandama.

Mots clés : Modélisation, Transport de sédiment, Embouchure, Fleuve Bandama. 


\section{Thème 2 - Dynamique sédimentaire}

\section{Introduction}

L'estuaire de Grand-Lahou représente, de par sa diversité écologique et environnementale, une importante source de subsistance et une source de revenu pour les populations riveraines dont la principale activité économique est la pêche traditionnelle (KONAN et al., 2013). Cependant, l'estuaire de Grand-Lahou subit des phénomènes sédimentaires qui ont pour conséquences la modification de la morphologie de l'estuaire, la migration de la passe d'Est en Ouest (WOGNIN et al., 2007) et la variation significative des paramètres physico-chimiques des plans d'eau (KONAN et al., 2009). Les tirants d'eau en lagune sont généralement faibles; ce qui rend la navigation assez difficile.

La pêche qui occupait naguère plus $60 \%$ des activités de la population connaît une baisse et on assiste donc à une paupérisation plus accentuée de la population de Grand Lahou (ECOUTIN et al., 1994).

Dans le but de redonner une vitalité à l'économie de la région, l'Etat ivoirien a construit une école de pêche à Grand-Lahou avec un port d'application et des bateaux pour les activités pratiques en eau continentale et en eau marine. Cependant, les bateaux de l'école de pêche ne peuvent pas accéder à la mer parce que la passe reste non navigable. Pour permettre aux bateaux de relier le port et la mer, un projet de construction d'un chenal reliant le port d'application de l'école de pêche à la mer est envisagé.

Pour faire, face aux problèmes hydrosédimentaires tels que ceux qui se produisent dans le système lagunaire de Grand-Lahou, plusieurs recherches développent des modèles numériques qui présentent de grands avantages dans la compréhension et la gestion des processus physiques liés à la dynamique des écoulements et au transport des sédiments (HUSSAM, 2010 ; MISBAH et al., 2014 ; JOONGU, 2015 ; AASTHA et al., 2016).

Ainsi, dans le but d'accompagner le projet de construction du chenal de Grand-Lahou, la présente étude se propose de modéliser le transport des sédiments à l'embouchure du fleuve Bandama. Cette étude s'appuie sur le modèle hydrodynamique réalisé par KOUASSI et al. (2016).

\section{Site et méthode}

\subsection{Site d'étude}

La zone estuarienne de Grand-Lahou est s'étend entre les latitudes $4^{\circ} 26$ et $5^{\circ} 20$ Nord et entre les longitudes $4^{\circ} 20$ et $5^{\circ} 20$ Ouest. Le système lagunaire de Grand-Lahou est constitué des lagunes Nyouzoumou, Tadjo, Mackey et Tagba. La lagune Tagba est le seul exutoire en mer de ce système lagunaire (Figure 1). 


\section{XVèmes Journées Nationales Génie Côtier - Génie Civil \\ La Rochelle, 29 au 31 mai 2018}

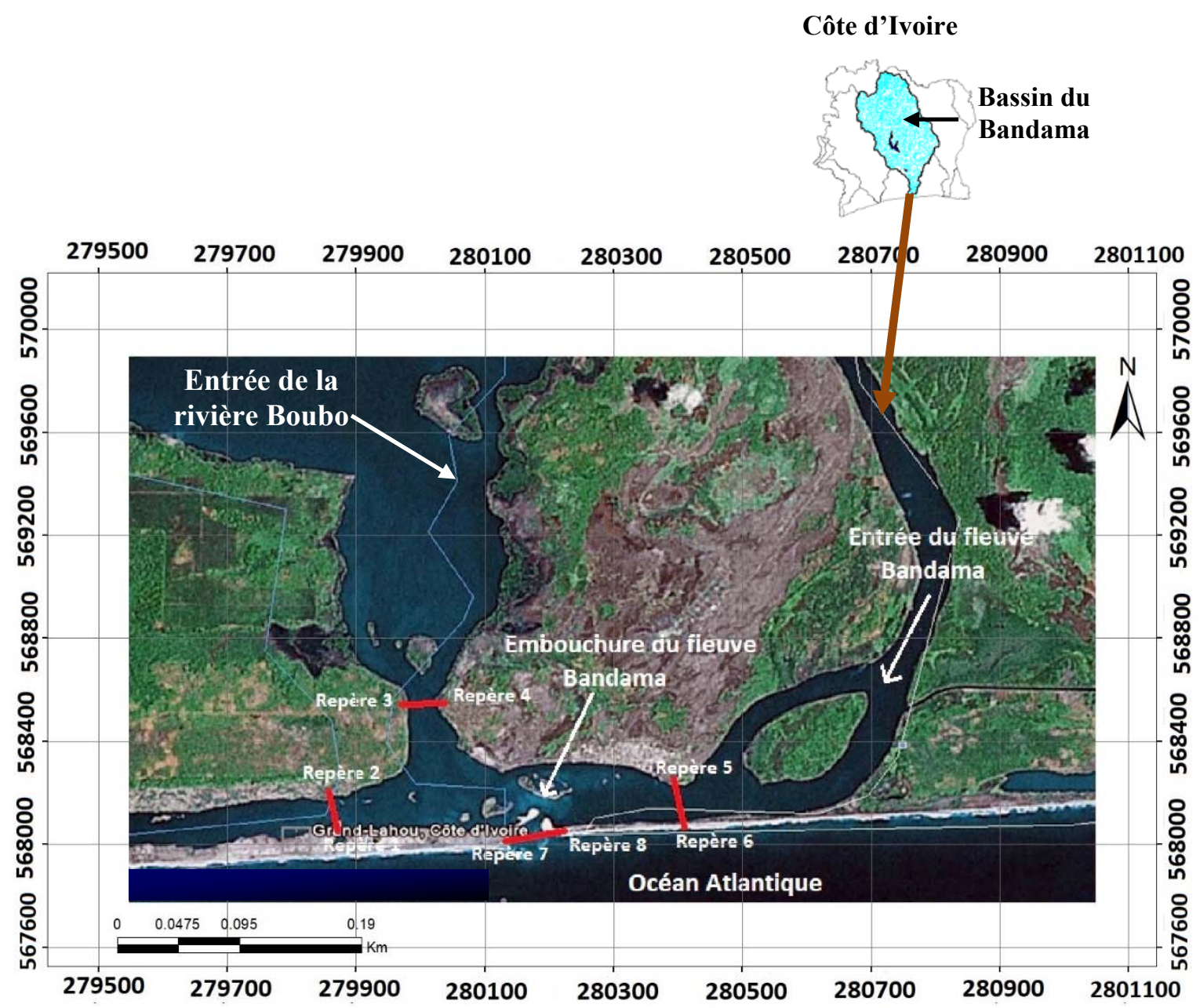

Figure 1. Localisation de la zone estuarienne de Grand-Lahou

L'hydrologie de l'estuaire est essentiellement sous l'influence des régimes du fleuve Bandama dominés par le régime tropical de transition du nord du bassin versant du fait de sa forme allongée, des cours d'eau côtiers et des saisons marines (WOGNIN, 2004). La dynamique sédimentaire au niveau de l'embouchure du fleuve Bandama se traduit par une érosion et une migration de la passe d'Est en Ouest (Figure 4). De même, il se pose des problèmes de navigation à cause des haut fonds (WOGNIN et al., 2008).

\subsection{Méthodes}

1.

\subsubsection{Acquisition des données}

Les caractéristiques hydrologiques (débits) du fleuve Bandama et de la rivière Boubo ont été obtenues auprès de la Direction de l'Eau du Ministère des Infrastructures Economiques de la Côte d'Ivoire. Les données pluviométriques de la ville de GrandLahou ont été acquises auprès de la Société d'Exploitation et de Développement Aéroportuaire, Aéronautique et Météorologique (SODEXAM). 


\section{Thème 2 - Dynamique sédimentaire}

Les données topographiques et bathymétriques utilisées dans la présente étude sont celles collectées en 2010 par l'entreprise DRAG AFRIK SA dans le cadre du projet de construction du chenal de Grand-Lahou. La zone des levés se situe entre $4^{\circ} 58^{\prime} 37^{\prime \prime}$ et $5^{\circ} 01^{\prime} 17^{\prime \prime}$ de longitude Ouest et $5^{\circ} 07^{\prime} 04^{\prime \prime}$ et 5 $5^{\circ} 09^{\prime} 19^{\prime \prime}$ de latitude Nord, représentant une surface de $05 \mathrm{~km}^{2}$ de plan d'eau et $01 \mathrm{~km}^{2}$ sur la terre ferme.

Les données de houle ont été obtenues auprès de l'United Kingdom Meteorological Office (UKMO) au point de de grille du maillage UKMO situé environ $65 \mathrm{~km}$ au SudOuest de Grand-Lahou. Des mesures de courants ont été réalisées à l'aide d'un courantomètre ADCP, Workhorse Rio Grande de RD Instruments. Les mesures ont été réalisées sur quatre (4) radiales (Figure 1).

Les sédiments ont été prélevés sur toute l'embouchure (Figure 2) pour déterminer les faciès granulométriques sédimentaires. Les sédiments dans la lagune sont dominés par les vases et les sables fins. Les sables moyens se rencontrent sur la plage. Des prélèvements d'eau ont également été réalisés dans la lagune pour déterminer les concentrations en matières en suspension.

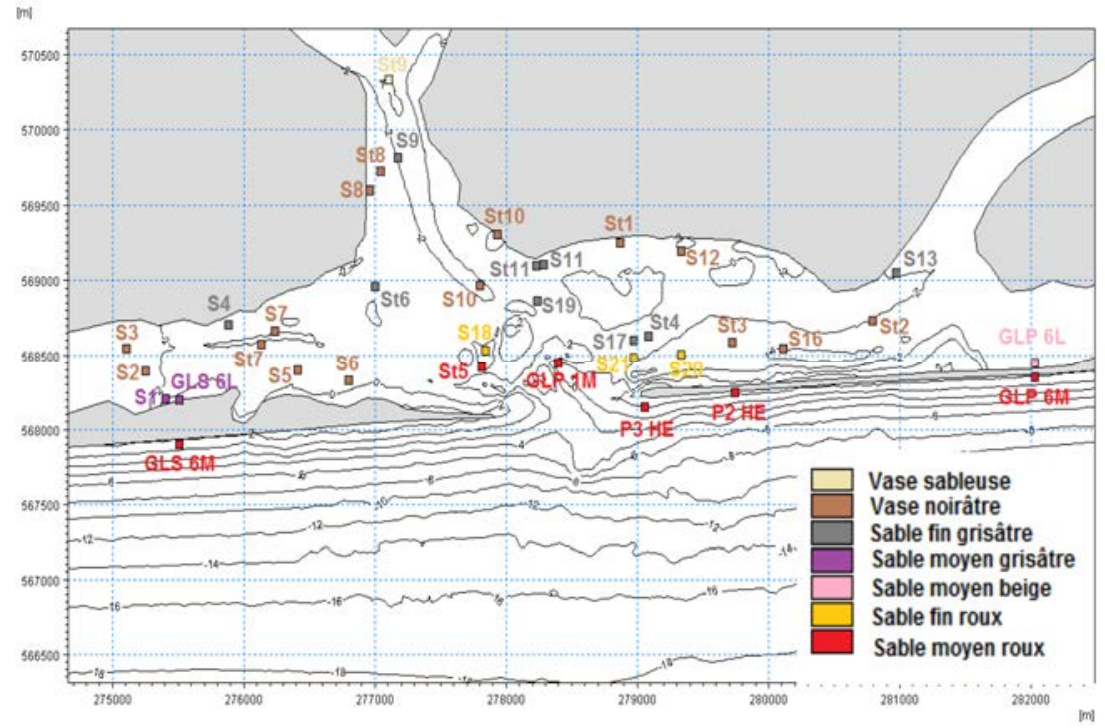

Figure 2. Distribution des faciès sédimentaires à l'embouchure du Bandama.

\subsubsection{Modélisation}

La modélisation du transport des sédiments a été entreprise dans la lagune avec le modèle MIKE 21 HD FM, en couplant le module MT (Mud Transport) au module hydrodynamique HD afin de simuler la dynamique des sédiments vaseux et des sables fins qui se fait principalement en suspension dans la colonne d'eau. Les équations aux dérivées partielles ci-dessous décrivent l'équation de la continuité, les équations de la conservation de quantité de mouvement intégrée sur les profondeurs d'eau et l'équation de transport des sédiments (DHI, 2007 ; KOUASSI et al., 2013). 


\section{XVèmes Journées Nationales Génie Côtier - Génie Civil \\ La Rochelle, 29 au 31 mai 2018}

$\frac{\partial \zeta}{\partial t}+\frac{\partial p}{\partial x}+\frac{\partial q}{\partial y}=0$

Quantité de mouvement dans la direction $\mathrm{x}$

$\frac{\partial p}{\partial}+\frac{\partial}{\partial x}\left(\frac{p^{2}}{h}\right)+\frac{\partial}{\partial y}\left(\frac{p \cdot q}{h}\right)+g h \frac{\partial \zeta}{\partial x}=-\frac{g \cdot p \sqrt{p^{2}+q^{2}}}{C^{2} h^{2}}+f \cdot V \cdot V_{X}-\frac{h}{\rho_{w}} \cdot \frac{\partial p_{a}}{\partial x}+\Omega q+\frac{1}{\rho_{w}}\left[\frac{\partial}{\partial x}\left(h \cdot \tau_{X X}\right)+\frac{\partial}{\partial y}\left(h \cdot \tau_{x y}\right)\right]$

Quantité de mouvement dans la direction y

$\frac{\partial q}{\partial t}+\frac{\partial}{\partial y}\left(\frac{q^{2}}{h}\right)+\frac{\partial}{\partial x}\left(\frac{p \cdot q}{h}\right)+g \cdot h \cdot \frac{\partial \zeta}{\partial y}=-\frac{g \cdot q \sqrt{p^{2}+q^{2}}}{C^{2} h^{2}}+f V V_{y}-\frac{h}{\rho_{w}} \cdot \frac{\partial p_{a}}{\partial y}-\Omega \cdot p+\frac{1}{\rho_{w}}\left[\frac{\partial}{\partial x}\left(h \cdot \tau_{x y}\right)+\frac{\partial}{\partial y}(h \cdot \tau y y)\right]$

Equation transport des sédiments

$\frac{\partial c}{\partial t}+u \frac{\partial c}{\partial x}+v \frac{\partial c}{\partial y}=\frac{1}{h} \frac{\partial}{\partial x}\left(h D x \frac{\partial c}{\partial x}\right)+\frac{1}{h} \frac{\partial}{\partial y}\left(h D y \frac{\partial c}{\partial y}\right)+Q_{L} C_{L} \frac{1}{h}-S$

\subsubsection{Conditions de forçage et paramétrage du modèle}

La marée dans l'Océan Atlantique et le débit du fleuve Bandama sont les principaux agents de forçage dans ce modèle hydrodynamique.

Les paramètres de calage pertinents dans cette étude sont les frottements sur le fond et le débit du fleuve Bandama. La dynamique de l'écoulement dans la baie lagunaire de Grand-Lahou a été appréciée sur un cycle de marée en utilisant un débit du fleuve Bandama de $220 \mathrm{~m}^{3} / \mathrm{s}$ pour le calage du modèle.

Un coefficient de rugosité Manning de $32\left(\mathrm{~m}^{1 / 3} / \mathrm{s}\right)$, en moyenne a été utilisé sur l'ensemble du modèle à part l'entrée de la lagune Tagba où un coefficient Manning de 10 a été imposé à cause de la présence des vases.

Le modèle de transport de sédiments a pris en compte deux fractions distinctes de particules : les vases de diamètre médian $10 \mu \mathrm{m}$ et les sables fins de diamètre médian $200 \mu \mathrm{m}$.

\section{Résultats}

\subsection{Bathymétrie de l'embouchure du Bandama}

La carte bathymétrique de l'embouchure du Bandama a été établie à partir de mailles dont la taille a été ajustée suivant la morphologie des fonds et la précision souhaitée. Ainsi, la taille des mailles varie de $10 \mathrm{~m}$ au niveau de l'embouchure à $1000 \mathrm{~m}$ au large. Le fond de la lagune est caractérisé par la présence de hauts fonds (Figure 3). 


\section{Thème 2 - Dynamique sédimentaire}

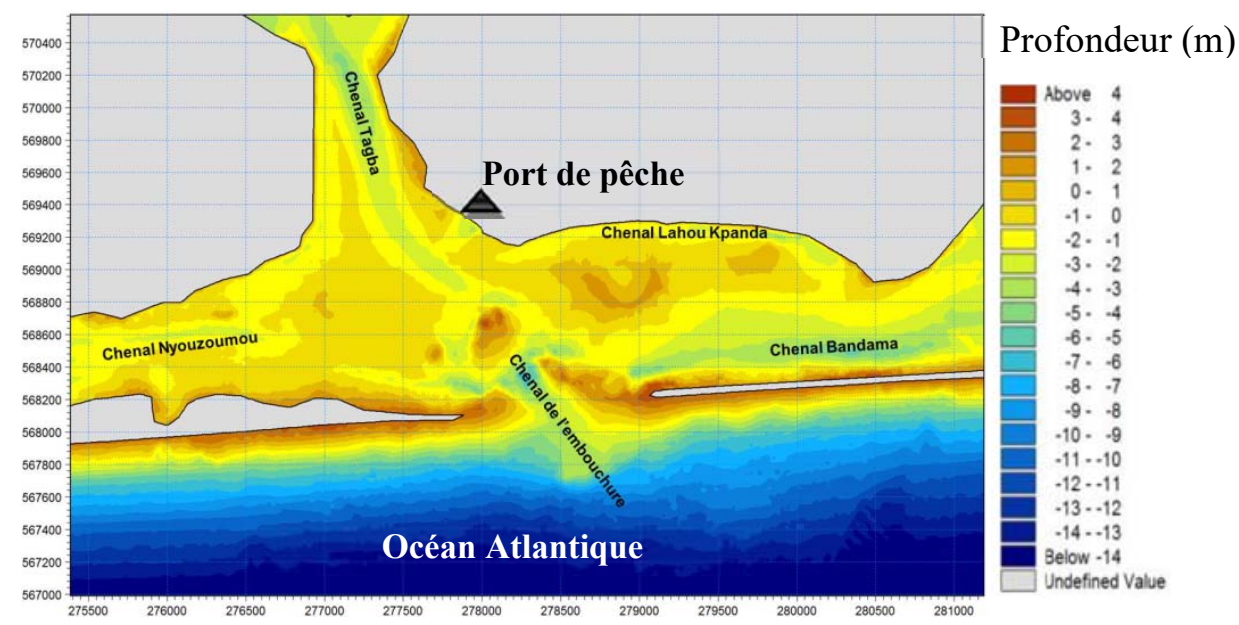

Figure 3. Bathymétrie de l'embouchure du fleuve Bandama (2010)

\subsection{Modèle hydrodynamique}

La marée à Grand-Lahou est semi-diurne à forte inégalité diurne : on observe chaque jour deux pleines mers de niveaux relativement proches, mais deux basses-mers de niveaux différents de l'ordre de $0,4 \mathrm{~m}$.

Sous l'effet des marées, la variation du niveau d'eau est accompagnée d'un changement de sens d'écoulement des eaux. L'écoulement se fait d'amont en aval durant les périodes de jusant et d'aval en amont durant les périodes de flot (Figures $4 \mathrm{a}$ et $4 \mathrm{~b}$ ).
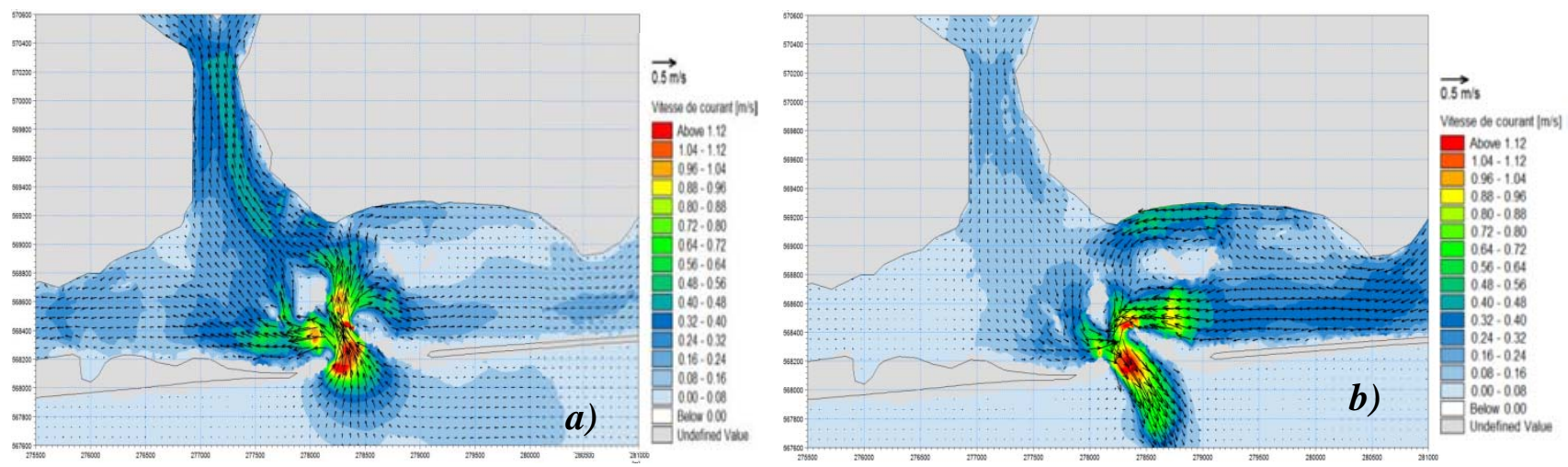

Figure 4. Champ d'écoulement modélisé au 30 juin 2012, (a) période de flot, 12:00 ;

(b) période de jusant, 17:00

\subsection{Modèle de transport des sédiments}

Les cartes des érosions-dépôts sur la période de simulation montrent qu'en période d'étiage, les particules de vases se déposent dans les chenaux du Bandama et de LahouKpanda, ainsi que sur les bancs en arrière des ilots (Figure 5). Le secteur Ouest (lagune de Nyouzoumou) est aussi le siège de sédimentation mais ce phénomène est fortement renforcé en période de crue, les concentrations en suspension étant plus élevées. 


\section{XVèmes Journées Nationales Génie Côtier - Génie Civil \\ La Rochelle, 29 au 31 mai 2018}
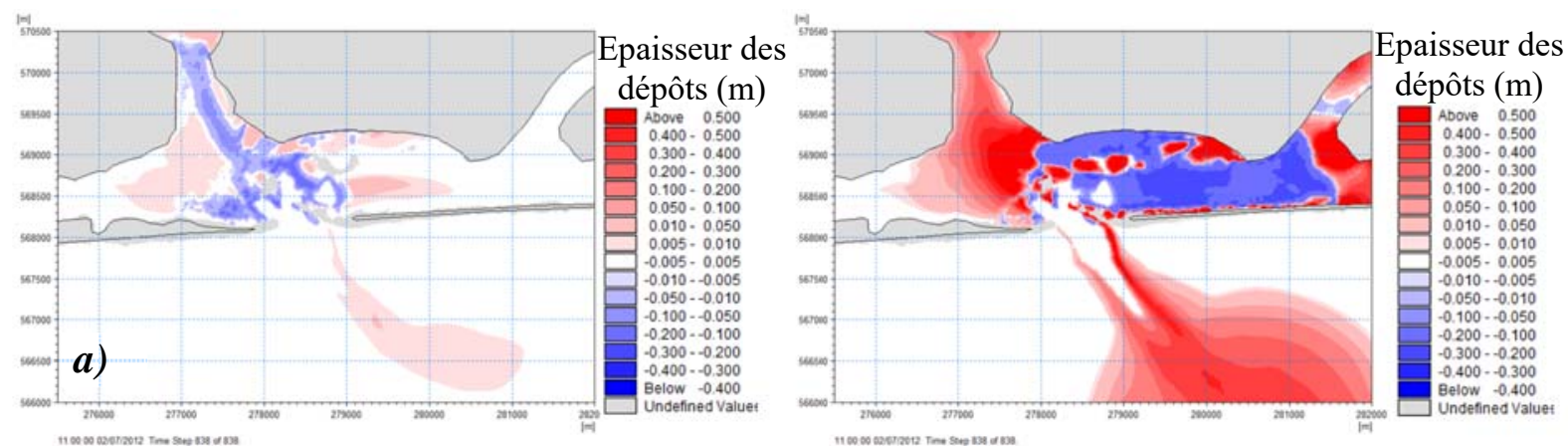

Figure 5. Tendance aux érosions-dépôts des vases au bout d'un mois de simulation-

(a) Conditions de débit d'étiage (75 m³ $/ \mathrm{s}$ ) et (b) conditions de crue maximale (1500 $\mathrm{m}^{3} / \mathrm{s}$ )

Les cartes d'érosions-dépôts des sables fins montrent qu'en condition d'étiage, les sables fins sont mobilisés par les courants de marée dans le secteur central, de part et d'autre de l'ilot central (Figure 6). On constate qu'une accumulation transversale au chenal apparait aux abords du port de pêche. En condition de crue moyenne, les sables sont transportés dans chenal de Lahou-Kpanda, et s'accumulent à la pointe, pouvant gêner également l'approche du port de pêche. A l'embouchure, les sables tendent à s'accumuler dans le secteur Sud-Est de la passe.
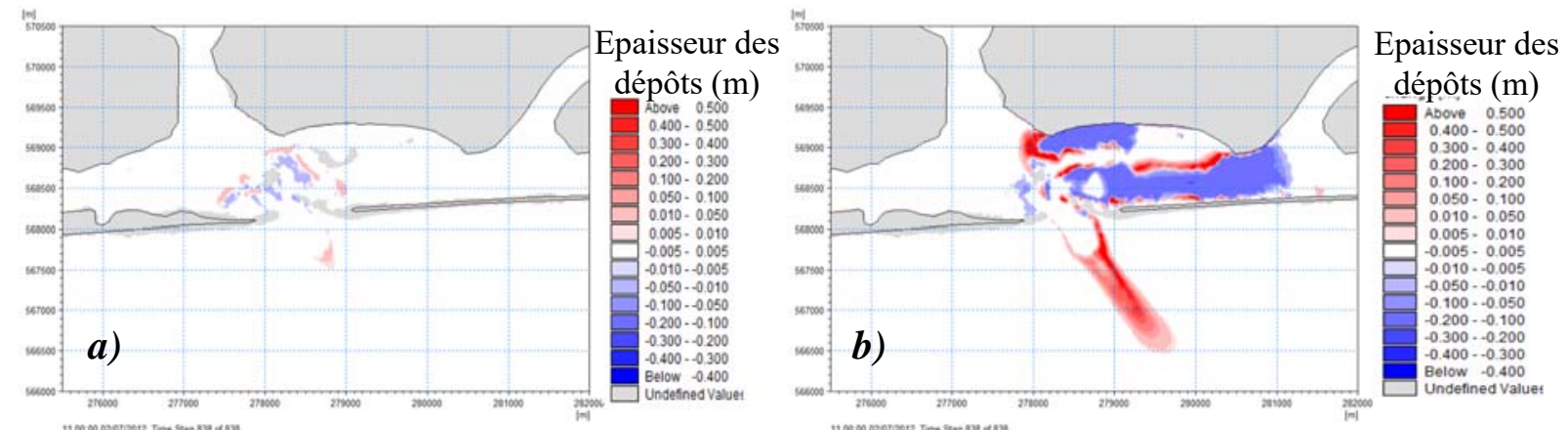

Figure 6. Tendance aux érosions-dépôts des sables fins au bout d'un mois de simulation (a) Conditions de débit d'étiage ( $\left.75 \mathrm{~m}^{3} / \mathrm{s}\right)$ et (b) condition de crue maximale (1500 $\left.\mathrm{m}^{3} / \mathrm{s}\right)$

\section{Discussion}

Les phénomènes hydrodynamiques à l'embouchure du fleuve Bandama sont sous l'influence du fleuve Bandama, de la rivière Boubo, du système lagunaire de GrandLahou et de l'Océan Atlantique. Le dynamisme du milieu est surtout contrôlé par les débits du Bandama et les flux océaniques.

Selon WOGNIN et al. (2008), la dynamique de marée dans l'estuaire du Bandama créerait un point nodal, zone d'accumulation des particules en suspension, dont la position varie en fonction du débit du Bandama. Par fort débit, le panache turbide est expulsé en mer, avec une tendance à s'évacuer vers l'Est, du fait de l'orientation des courants de jusant dans la passe et des courants sur le plateau continental. 


\section{Thème 2 - Dynamique sédimentaire}

Malgré la migration de l'embouchure due aux mouvements hydro-sédimentaires et à la dérive littorale, l'embouchure du Bandama reste toujours ouverte contrairement à celle du fleuve Comoé tel que rapporté par ADOPO et al. (2008). Cela peut être dû au fait que les débits du Bandama sont relativement suffisants pour pénétrer dans l'Océan Atlantique pendant les périodes de jusant et surtout pendant les crues du fleuve.

Les résultats obtenus du modèle de transport sont en accord avec les observations de terrain. En période d'étiage, les vases tendent à se déposer dans les chenaux du Bandama et de Lahou-Kpanda, alors qu'elles sont évacuées en période de crue.

En période de crue, les forts courants tendent à éroder les chenaux du Bandama et de Lahou-Kpanda, générant un transport vers l'Ouest. On observe une tendance à l'accumulation au sud du port de pêche.

\section{Conclusion}

La modélisation du transport des sédiments a été réalisée dans la lagune avec le modèle MIKE 21 HD FM, en couplant le module MT (Mud Transport) au module hydrodynamique HD a permis de simuler la dynamique des sédiments vaseux et des sables fins de l'embouchure du Bandama.

Les résultats sont cohérents avec la répartition des sédiments et la dynamique des particules fines à l'embouchure de Grand-Lahou.

En période d'étiage, les vases tendent à se déposer dans les chenaux du Bandama et de Lahou-Kpanda, alors qu'elles sont évacuées en période de crue.

La dynamique des sables fins est limitée au secteur central en période d'étiage, soumis à l'action des courants de marée. En période de crue, les forts courants tendent à éroder les chenaux du Bandama et de Lahou-Kpanda, générant un transport vers l'Ouest. On observe une tendance à l'accumulation au sud du port de pêche.

\section{Remerciements}

La réalisation de cette étude a été soutenue par le Groupe DHI à qui nous adressons nos sincères remerciements pour leur contribution à nos travaux.

\section{Références bibliographiques}

ADOPO K.L., KOUASSI K.L., WOGNIN A.V.I., MONDE S., MÉLÈDJE N.H. AKA K. (2008). Characterization of the sediments and morphology of the Comoé river mouth (Grand-Bassam, Côte d'Ivoire). Revue Paralia, n, pp 2.1-2.10. https://doi.org/10.5150/revueparalia.2008.002

AASTHA C., RIJAN B.K., AHUTI S. (2016). Assessment of Sediment Load of Langtang River in Rasuwa District, Nepal. Journal of Water Resource and Protection, Vol.8, pp 84-92. https://doi.org/10.4236/jwarp.2016.81007 


\section{XVèmes Journées Nationales Génie Côtier - Génie Civil La Rochelle, 29 au 31 mai 2018}

DHI (Danish Hydraulic Institute) (2007). Coastal hydraulic and oceanography, hydrodynamic module. Scientific documentation, 53p.

ECOUTIN J.M., DURAND J.R., LAE R., HIÉ J.P. (1994). L'exploitation des stocks, In environnement des ressources aquatiques de Côte d'Ivoire, les milieux lagunaires, Tome II. pp 339-444.

HUSSAM AL-R. (2010). Transport of Suspended Solids in Dammam (Saudi Arabia) Coastal Areas: Fish Market Works. Journal of Water Resource and Protection, Vol. 2, pp 540-544. https://doi.org/10.4236/jwarp.2010.26061

JOONGU K., HONGKOO Y. (2015). Survey and Analysis of the Sediment Transport for River Restoration: The Case of the Mangyeong River. Open Journal of Civil Engineering, Vol. 5, pp 399-411. https://doi.org/10.4236/ojce.2015.54040

KONAN K.S., KOUASSI A.M, ADINGRA A.A., GNAKRI D. (2009). Spatial and temporal variation of fecal contamination indicators in Graand-Lahou lagoon, Côte d'Ivoire. Journal of Applied Biosciences, Vol. 23, pp 1422-1435.

KONAN K.S., KOUASSI K.L., KOUAMÉ K.I., KOUASSI A.M., GNAKRI D. (2013). Hydrologie et hydrochimie des eaux dans la zone de construction du chenal du port de pêche de Grand-Lahou, Côte d'Ivoire. Int. J. Biol. Chem. Sci., Vol. 7, n² 2, pp 819-831. https://doi.org/10.4314/ijbcs.v7i2.37

KOUASSI K.L., DEME M., KONAN K.S., YAHIRI B.P., KONÉ D., SANCHEZ M., AKA K. (2016). Modélisation du fonctionnement hydrodynamique de l'embouchure du fleuve Bandama, Grand-Lahou, Côte d'Ivoire. Acte des XIV ${ }^{\text {èmes }}$ Journées Nationales Génie Côtier - Génie Civil. pp 219-228. https://doi.org/10.5150/jngcgc.2016.025

KOUASSI K.L., KOUAMÉ K.I., KONAN K.S., SANCHEZ ANGULO M., DEMÉ M., MÉLÈDJE N.H. (2013). Two-Dimensional Numerical Simulation of the HydroSedimentary Phenomena in Lake Taabo, Côte d'Ivoire. Water Resources Management, Vol. 27, $\mathrm{N}^{\circ} 12$, pp 4379-4394. https://doi.org/10.1007/s11269-013-0417-x

MISBAH U., JAHIR B.A., ZAHIRUL H.K., G.M. JAHID H., TAUHIDUR R. (2014). Two Dimensional Hydrodynamic Modelling of Northern Bay of Bengal Coastal Waters. Computational Water, Energy, and Environmental Engineering, Vol. 3, pp 140-151. https://doi.org/10.4236/cweee.2014.34015

WOGNIN A. V. I. (2004). Caractérisation hydrologique et sédimentologique de l'embouchure du fleuve Bandama. Thèse de doctorat, Univ. de Cocody, 198p.

WOGNIN A.V., MONDE S., AFFIAN K., COULIBALY A., AKA K. (2007). Modèle de circulation des eaux dans l'estuaire du fleuve Bandama en Côte d'Ivoire. Sud Sciences \& Technologies, Vol. 15, pp 5-12.

WOGNIN V., MONDE S., COULIBALY A., KOUASSI K.L., ADOPO L., AFFIAN K. AKA K. (2008). Waters Model Circulation in the Estuary of Bandama. Rivers Flows and Tide condition's Incidence. European Journal of Scientific Research, Vol. 19(2), pp 304-314. 
Thème 2 - Dynamique sédimentaire 